\title{
Immune system plays an important role in the success and failure of conventional cancer therapy
}

\section{"...the unseen contribution of endogenous immunity should be considered when testing therapies in preclinical models."}

There are a multitude of different drugs available to kill cancer cells. While there has been an iterative improvement in the development of models to test drugs, there is a limited correlation between preclinical success and clinical success [1]. Preclinical studies of new drugs focus on cell lines and xenografted human tumors in immunodeficient mice and, thus, ignore the contribution of the immune system. The field of cancer drug development has moved towards targeted inhibitors that focus on key signal transduction pathway molecules such as Erk and Akt, which are important in cancer cell survival. It is of concern to immunologists that these are also critically important regulators of immune cells.

\section{"The field of cancer drug development has moved towards targeted inhibitors that focus on key signal transduction pathway molecules such as Erk and Akt, which are important in cancer cell survival."}

The majority of solid tumors grow in immunecompetent patients. A critical stage in the evolution of a tumor to the point where it presents as a symptomatic mass requires the control of hostimmune responses. CD8 $\mathrm{T}$ cells specific for tumor antigens are identifiable in patients with many types of cancer, indicating that the immune system is capable of recognizing tumors. Tumors arise more readily in immunodeficient animals, but are less equipped to resist adaptive immune control [2]. This implies that, at presentation, advanced cancers in otherwise normal patients have some degree of control over their local immune environment. Nonetheless, current treatment decisions are not influenced by the presence or absence of an antitumor immune response.

While cancers can systemically perturb immune systems, the majority of patients sustain the ability to clear opportunistic infections and show few functional signs of systemic adaptive immune deficiency, such that even multiple-treated, late-stage cancer patients can be vaccinated. Instead it appears that tumors have a predominately locoregional effect on the immune system with increasing systemic effect with progressive tumor burden. Tumor macrophages appear to be critical in regulating this local immune environment in cancer, and this has consequences for patients. Tumors that present with a high-macrophage infiltrate, with a poor T-cell infiltrate, or with a high macrophage:CD8 ratio have a poorer prognosis [3-5]. While the importance of the immune system to cancer therapy is not a difficult sell to immunologists, chemotherapy, surgery and radiation therapy are commonly considered immunosuppressive interventions. However, amongst patients receiving these treatments, the local immune environment matters. Cancer patients with an impaired immune response develop metastases faster following surgery, radiation and chemotherapy [6]. In a murine model, depletion of CD8 $T$ cells increased local recurrence following surgery [7]; by contrast, stimulating the endogenous immune response at the time of the operation has been shown to decrease local recurrence $[7,8]$. Similarly, in animal models, the efficacy of radiation therapy is partly dependent on functional T-cell responses [7,9], and radiation therapy can be made more effective by improving T-cell immune responses $[7,9,10]$. Thus, the local immune environment within a tumor may play a critical role in the success or failure of conventional therapies and should be considered when designing and developing new therapies. We propose that the stromal environment of the tumor can protect those small numbers of cells that escape cytotoxic and surgical therapies. This stromal environment is dictated by the polarized myeloid cells in the tumor, and in turn myeloid polarization is influenced by adaptive immune cells. Since the sum total of the tumor immune environment is influential, the unseen contribution of endogenous immunity should be considered when testing therapies in preclinical models.

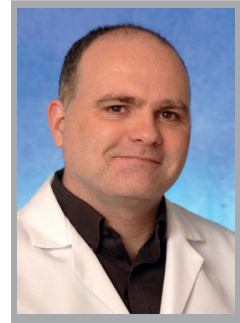

Michael J Gough

Author for correspondence: Earle A Chiles Research Institute, Robert W Franz Cancer Research Center, Providence Cancer Center Portland, OR 97213, USA Tel.: +1 5032153928 michael.gough@providence.org

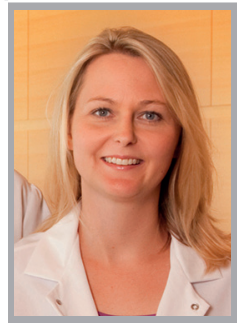

Marka R Crittenden The Oregon Clinic, Portland, OR 97213, USA 
Multiple layers of evidence exist regarding the role of the endogenous immune system in tumorigenesis, tumor progression and tumor resistance to therapy. Spontaneous and chemically-induced tumors, which may accurately model the progressive stages of carcinogenesis, are highly influenced by the host immune system. Chemically-induced tumors require chronic proinflammatory conditions, including expression of the proinflammatory cytokine TNF- $\alpha$ for carcinogenesis [11]. By contrast, progression to invasive cancer and metastatic spread is independent of TNF- $\alpha$ [11], but is dependent on macrophage production of the inflammatory resolution-phase cytokine VEGF [12]. Elevated VEGF expression is associated with the emergence of the characteristic vascular loops and sprouts of angiogenic neovasculature in developing tumors, and results in an elevated interstitial pressure compared with normal tissues [13]. This elevated interstitial pressure results in poor drug perfusion, despite leaky vasculature, and is one of the factors contributing to the many log-fold difference in efficacy of chemotherapy in vitro and in vivo [14]. VEGF inhibition results in decreased interstitial pressure concomitant with fewer immature vessels or 'vascular normalization' [15]. Therefore an important advantage of targeting VEGF may be in better chemotherapy perfusion into the tumor. Olive et al. demonstrated that targeting the tumor stroma with inhibitors of hedgehog signaling resulted in vascular remodeling, increased drug penetration and increased the efficacy of chemotherapy in murine models [16] These data demonstrate that the tumor stroma actively protects cancer cells from conventional therapy, and we propose that the development of a negative tumor stroma is directed by immune infiltrates into the tumor site.

There are good data implicating tumor macrophages in dictating the stromal environment of the tumor. Macrophage activation may be broadly split into classical (M1) activation, associated with proinflammatory responses, versus alternative (M2) activation, associated with inflammatory resolution and wound healing. Tumors have famously been described as 'wounds that do not heal' [17], and a gene expression profile that provides a 'wound-response signature' is predictive of local recurrence following breast-conserving surgery in breast cancer patients [18]. Such wound healing immune responses are characteristically associated with suppression of adaptive immune responses. M2 macrophages express a range of molecules associated with immune suppression, including arginase, IL-10 and TGF- $\beta$. Moreover, it is the constant proresolution and prowound healing environment caused by M2 tumor macrophages that combines to drive angiogenesis and thereby resistance to treatment, but also invasion and metastases [12]. Recent data demonstrated that immunotherapy with antibodies to CD40 improved the predicted effect of chemotherapy in pancreatic cancer patients [19]. Surprisingly, the mechanism did not occur via T-cell stimulation, but instead via anti-CD40-bound macrophages before their migration into the tumor, and resulted in a transient systemic surge in IFN- $\gamma$ and TNF- $\alpha$, but not IL-10, suggestive of M1 repolarization [19]. These data demonstrate that myeloid differentiation probably underpins many of the tumor features that limit effective therapy and represents an important potential target in cancer patients.

\section{"The reality is that endogenous immune mechanisms already dictate how some patients respond to conventional therapy."}

It is important to note that cytokines derived from adaptive immune cells are the most potent agents directing macrophage polarization. We propose that the adaptive immune system is complicit in the development of the immune environment of tumors. CD4 T cells have been shown to contribute to tumor invasion and metastases by driving the late-stage M2 differentiation of tumor macrophages, via secretion of IL-4 [20]. However, T cells are also an important source of IFN- $\gamma$, which is a key cytokine for the M1 activation of macrophages. Repeated administration of tumor-specific effector $\mathrm{T}$ cells can remodel the tumor environment, resulting in reduced angiogenesis and normalization of the tumor vasculature [21]. Importantly, studies in colorectal cancer patients have shown that an IFN- $\gamma$ gene signature and VEGF represent opposing predictors of recurrence [22]. Thus, while the primary intent of T-cell immunotherapy is to provide cell-mediated cytotoxicity to cancer cells, it is also possible that T-cell responses have the opportunity to enhance cytotoxic therapies through changes to the tumor environment, for example via local IFN- $\gamma$ secretion. Cytotoxic $\mathrm{T}$ cells can eliminate tumors where they are unable to directly kill cancer cells, by targeting the tumor stroma [23]. Radiation and chemotherapy have been shown to cause transfer of antigens from cancer cells to macrophages in the tumor during a short window following cytotoxic therapy [24], and made these cells targets for tumor antigen-specific cytotoxic T cells. Stromal targeting was necessary for complete elimination of tumors where tumor antigen presentation was suboptimal [23], and required IFN- $\gamma$ production 
by $\mathrm{T}$ cells and the IFN- $\gamma$ receptor on macrophages and other stromal cells in the tumor [25]. We interpret these data to suggest that tumors may establish a permissive treatment environment, with IFN- $\gamma$, CD8 T cells and M1 differentiation of macrophages, versus a resistant tumor environment, with IL-4, angiogenesis and M2 differentiation of macrophages.

This dichotomy represents an opportunity for immunotherapy. The vast majority of the cancer immunotherapy literature has studied techniques to increase tumor antigen-specific T-cell control of cancer cells. Unfortunately, immunotherapies alone have had limited success in the treatment of advanced or established cancers. Part of this limitation may be the limited ability of effector $T$ cells to penetrate and function in the M2-dominated suppressive tumor environment. However, adaptive immunity can transiently change the tumor. Therapy with agonistic antibodies to the T-cell costimulatory molecule CD134 resulted in an increased influx of CD8 $\mathrm{T}$ cells into established murine tumors and, importantly, caused a simultaneous decrease in tumor macrophages, reducing immune suppression by those macrophages remaining [26]. Similarly, therapy with blocking antibodies to the negative regulatory molecule CD152 causes T-cell influxes to the tumor in mice [27] and in patients [28]. Thus, it is possible that immunotherapies, while limited in their efficacy as solo agents, may change the tumor environment to make conventional therapies more effective. We propose that adaptive immunity has the capacity to induce inflammatory-mediated changes in the tumor stroma, particularly via effects on tumor macrophages.

In conclusion, we believe that there is considerable scope for trials of immunotherapy in combination with conventional therapy. The reality is that endogenous immune mechanisms already dictate how some patients respond to conventional therapy. We are only beginning to understand the immune component of conventional therapies, but there may already be many existing immunotherapies, ruled ineffective as single agents, that are capable of manipulating the tumor toward a favorable immune environment and could be effective in combination therapies. In addition, many targeted therapies that are ruled effective in preclinical models using xenograft testing may fail to adequately address the immune system's role in the control of tumors following conventional therapies and this may explain in part the disparate effects seen when these agents transition into clinical trials.

\section{Financial \& competing interests disclosure}

The authors have no relevant affiliations or financial involvement with any organization or entity with a financial interest in or financial conflict with the subject matter or materials discussed in the manuscript. This includes employment, consultancies, honoraria, stock ownership or options, expert testimony, grants or patents received or pending, or royalties.

No writing assistance was utilized in the production of this manuscript.

\section{References}

1 Johnson JI, Decker S, Zaharevitz D et al. Relationships between drug activity in NCI preclinical in vitro and in vivo models and early clinical trials. Br. J. Cancer 84(10), 1424-1431 (2001).

2 Shankaran V, Ikeda H, Bruce AT et al. IFN $\gamma$ and lymphocytes prevent primary tumour development and shape tumour immunogenicity. Nature 410(6832), 1107-1111 (2001).

3 Leek RD, Lewis CE, Whitehouse R, Greenall M, Clarke J, Harris AL.

Association of macrophage infiltration with angiogenesis and prognosis in invasive breast carcinoma. Cancer Res. 56(20), 4625-4629 (1996).

4 DeNardo DG, Brennan DJ, Rexhepaj E et al. Leukocyte complexity predicts breast cancer survival and functionally regulates response to chemotherapy. Cancer Discov. 1(1), 54-67 (2011).
5 Galon J, Costes A, Sanchez-Cabo F et al.

Type, density, and location of immune cells within human colorectal tumors predict clinical outcome. Science 313(5795), 1960-1964 (2006).

6 Apetoh L, Ghiringhelli F, Tesniere A et al. Toll-like receptor 4-dependent contribution of the immune system to anticancer chemotherapy and radiotherapy. Nat. Med. 13(9), 1050-1059 (2007).

7 Gough MJ, Crittenden MR, Sarff M et al. Adjuvant therapy with agonistic antibodies to CD134 (OX40) increases local control after surgical or radiation therapy of cancer in mice. J. Immunother. 33(8), 798-809 (2010).

8 Kwon ED, Foster BA, Hurwitz AA et al. Elimination of residual metastatic prostate cancer after surgery and adjunctive cytotoxic T lymphocyte-associated antigen 4 (CTLA-4) blockade immunotherapy. Proc. Natl Acad. Sci. USA 96(26), 15074-15079 (1999).
$9 \quad$ Lee Y, Auh SL, Wang Y et al. Therapeutic effects of ablative radiation on local tumor require $\mathrm{CD}^{+} \mathrm{T}$ cells: changing strategies for cancer treatment. Blood 114(3), 589-595 (2009).

10 Demaria S, Kawashima N, Yang AM et al. Immune-mediated inhibition of metastases after treatment with local radiation and CTLA-4 blockade in a mouse model of breast cancer. Clin. Cancer Res. 11(2 Pt 1), 728-734 (2005).

11 Moore RJ, Owens DM, Stamp G et al. Mice deficient in tumor necrosis factor- $\alpha$ are resistant to skin carcinogenesis. Nat. Med. 5(7), 828-831 (1999).

12 Lin EY, Li JF, Bricard G et al. Vascular endothelial growth factor restores delayed tumor progression in tumors depleted of macrophages. Mol. Oncol. 1(3), 288-302 (2007).

13 Boucher Y, Leunig M, Jain RK. Tumor angiogenesis and interstitial hypertension. Cancer Res. 56(18), 4264-4266 (1996). 
14 Curti BD, Urba WJ, Alvord WG et al. Interstitial pressure of subcutaneous nodules in melanoma and lymphoma patients: changes during treatment. Cancer Res. 53(Suppl. 10), S2204-S2207 (1993).

15 Tong RT, Boucher Y, Kozin SV, Winkler F, Hicklin DJ, Jain RK. Vascular normalization by vascular endothelial growth factor receptor 2 blockade induces a pressure gradient across the vasculature and improves drug penetration in tumors. Cancer Res. 64(11), 3731-3736 (2004).

16 Olive KP, Jacobetz MA, Davidson CJ et al. Inhibition of hedgehog signaling enhances delivery of chemotherapy in a mouse model of pancreatic cancer. Science 324(5933), 1457-1461 (2009).

17 Dvorak HF. Tumors: wounds that do not heal. Similarities between tumor stroma generation and wound healing. N. Engl. J. Med. 315(26), 1650-1659 (1986).

18 Nuyten DS, Kreike B, Hart AA et al. Predicting a local recurrence after breastconserving therapy by gene expression profiling. Breast Cancer Res. 8(5), R62 (2006).
19 Beatty GL, Chiorean EG, Fishman MP et al. CD40 agonists alter tumor stroma and show efficacy against pancreatic carcinoma in mice and humans. Science 331(6024), 1612-1616 (2011).

20 DeNardo DG, Barreto JB, Andreu P et al. $\mathrm{CD}^{+} \mathrm{T}$ cells regulate pulmonary metastasis of mammary carcinomas by enhancing protumor properties of macrophages. Cancer Cell 16(2), 91-102 (2009).

21 Ganss R, Ryschich E, Klar E, Arnold B, Hammerling GJ. Combination of T-cell therapy and trigger of inflammation induces remodeling of the vasculature and tumor eradication. Cancer Res. 62(5), 1462-1470 (2002).

22 Camus M, Tosolini M, Mlecnik B et al. Coordination of intratumoral immune reaction and human colorectal cancer recurrence. Cancer Res. 69(6), 2685-2693 (2009).

23 Spiotto MT, Rowley DA, Schreiber H. Bystander elimination of antigen loss variants in established tumors. Nat. Med. 10(3), 294-298 (2004).
24 Zhang B, Bowerman NA, Salama JK et al. Induced sensitization of tumor stroma leads to eradication of established cancer by T cells. J. Exp. Med. 204(1), 49-55 (2007).

25 Zhang B, Karrison T, Rowley DA, Schreiber $\mathrm{H}$. IFN- $\gamma$ - and TNF-dependent bystander eradication of antigen-loss variants in established mouse cancers. J. Clin. Invest. 118(4), 1398-1404 (2008).

26 Gough MJ, Ruby CE, Redmond WL, Dhungel B, Brown A, Weinberg AD. OX40 agonist therapy enhances CD8 infiltration and decreases immune suppression in the tumor. Cancer Res. 68(13), 5206-5215 (2008).

27 Yang YF, Zou JP, Mu J et al. Enhanced induction of antitumor $\mathrm{T}$-cell responses by cytotoxic $T$ lymphocyte-associated molecule- 4 blockade: the effect is manifested only at the restricted tumor-bearing stages. Cancer Res. 57(18), 4036-4041 (1997).

28 Huang RR, Jalil J, Economou JS et al. CTLA4 blockade induces frequent tumor infiltration by activated lymphocytes regardless of clinical responses in humans. Clin. Cancer Res. 17(12), 4101-4109 (2011). 